\title{
Gerações Y e Z no Stricto Sensu em Contabilidade e seus Valores Relativos ao Trabalho
}

\author{
Rayane Camila da Silva Sousa \\ https://orcid.org/0000-0002-7181-1607
}

Romualdo Douglas Colauto

https://orcid.org/0000-0003-3589-9389

\section{Resumo}

Objetivo: $\mathrm{O}$ estudo tem por objetivo verificar as diferenças de valores relativos ao trabalho de estudantes do stricto sensu em Contabilidade das Gerações Y e Z, considerando as características sociodemográficas. Método: Participaram da pesquisa 337 estudantes que em 2019 estavam matriculados em cursos de pósgraduação stricto sensu na área de Contabilidade. O estudo utiliza a Escala Revisada de Valores Relativos ao Trabalho, Análise Fatorial Exploratória, testes não paramétricos e de comparação emparelhada.

Resultados: Estudantes do gênero feminino atribuíram maior importância aos valores relacionados à estabilidade no trabalho e independência financeira, quando comparados aos estudantes do gênero masculino. Igualmente, foram encontradas diferenças significativas entre os grupos que compuseram as variáveis "responsabilidade financeira em casa", "experiência docente", "nível de escolaridade da figura materna" e "auxílio financeiro durante a pós-graduação".

Contribuições: $\mathrm{O}$ estudo viabiliza uma melhor compreensão das prioridades das Gerações Y e Z no ambiente de trabalho. A compreensão desses valores, possibilita discussões entre os próprios indivíduos sobre suas escolhas, carreiras, motivações e interesses. Esses aspectos são de grande importância para promover a satisfação do indivíduo no ambiente de trabalho e consequentemente o desempenho da instituição em que trabalham.

Palavras-chave: Valores Relativos ao Trabalho, Gerações Y e Z; Stricto Sensu; Contabilidade. 


\section{Introdução}

Os valores relativos ao trabalho são apontados como fundamentais no desenvolvimento de estudos sobre a temática laboral, visto que eles revelam as preferências dos indivíduos (Dose, 1997). O conceito de valor do trabalho passou por intensa transformação quando comparado a outros conceitos da vida cotidiana (Lévy-leboyer, 1994). A pesquisa do grupo MOW (1987) identificou que as pessoas trabalham em busca de remuneração, reconhecimento social e relacionamento interpessoal e que os principais elementos considerados como importantes no ambiente de trabalho são: autonomia, organização, ambiente social agradável, liberdade e poder (Blanch, 2007).

Os valores do trabalho têm sido discutidos como variáveis que influenciam a gestão de pessoas e o comportamento nas organizações (Cammarosano, Santos \& Rojas, 2014). A pluralidade de elementos atrelados a essas variáveis amplia as pesquisas sobre os valores do trabalho na área de gestão de pessoas, uma vez que, por meio dos valores, pode-se compreender o que é considerado importante pelos colaboradores no ambiente organizacional (Porto \& Tamayo, 2008; Cammarosano et al., 2014). Em razão disso, a temática representa um cenário propício para a compreensão de aspectos comportamentais e de ações subjetivas e produtivas da atividade laboral, as quais viabilizam o desenvolvimento de estratégias voltadas para a seleção e a retenção de pessoas alinhadas às diretrizes de cada organização (Zanelli, Borges-Andrade, \& Bastos, 2014).

Cavazotte, Lemos e Viana (2012) expõem que os valores relativos ao trabalho podem se diferenciar em função do contexto sociocultural e em função do perfil sociodemográfico. Nesse sentido, as gerações, consideradas um fator demográfico, também exercem influência no ambiente de trabalho em virtude de suas características e comportamentos que podem impactar nas dinâmicas de grupo e nos relacionamentos interpessoais (Cordeiro, 2012; Parry \& Urwin, 2011).

Sabe-se que as Gerações Y (1979-1992) e Z (a partir de 1993) foram influenciadas pelas mudanças tecnológicas ocorridas a partir dos anos 2000 (Twenge \& Campbell, 2008; Twenge, Campbell \& Freeman, 2012). Pessoas da Geração Y esperam que os ambientes de trabalho sejam diferentes do que os que os pais viveram; buscam ter o seu próprio negócio ou trocam o trabalho atual por outro que proporcione melhor qualidade de vida e ascensão na carreira, mesmo que isso implique em um salário menor (Moreira \& Araújo, 2018). A Geração $Z$ é mais cautelosa e impaciente que a Y devido ao ambiente de recessão econômica em que cresceram. Apesar disso, na Geração $Z$ as ambições são direcionadas para a criação da sua própria start-up, preferência por organizações horizontais à hierarquia e o desejo de transformar seu hobby em trabalho (Ozkan \& Solmaz, 2015; Grubb, 2016; Revista Exame, 2017).

Em específico, a inserção das Gerações (Y e Z) no ambiente de trabalho representa um dos principais desafios para os gestores, devido às características distintas que essas apresentam em relação a de gerações anteriores (Smola \& Sutton, 2002; Twenge \& Campbell, 2008; Furucho, Oswaldo, Graziano \& Elias Spers, 2015). Tais características apenas podem ser compreendidas, a partir do conjunto de crenças, valores e prioridades que formam cada geração.

Compreender as características e valores das gerações requer analisar data cronológica, fatos históricos, culturais e sociais. Estudos de cunho sociológico indicam haver diferenças de classes, gênero, étnico-raciais e culturais entre as gerações (Weller, 2010).

Mannheim (1993) acrescenta que as gerações devem ser analisadas de forma multidimensional, considerando suas relações com o meio social. Assim, estudar os valores relativos no trabalho é importante para compreender como as diferentes gerações se relacionam no ambiente de trabalho e como constroem seus valores (Comazzetto, Perrone, Vasconcellos \& Gonçalves, 2016). 
$\mathrm{Na}$ área contábil, o recrutar pessoas que se encaixam nas expectativas dos empregadores da área parece ser um grande desafio (Holt, Burke-Smalley \& Jones, 2017; Almeida \& Silva, 2018). As transformações significativas que o cenário contábil passou nas últimas décadas, aliadas a mudanças de padrões, aumento de regulamentação, inovações tecnológicas, concorrência virtual e stress laboral (Almeida, 2020) reforçam a necessidade de compreender se as novas gerações de estudantes estão se preparando para a realidade do trabalho profissional, assim como reforçam a necessidade de refletir se as empresas estão atentas às expectativas dos trabalhadores dessas gerações. Diante disso, o estudo se preocupa em responder a seguinte questão de pesquisa: quais as diferenças de valores relativos ao trabalho de estudantes do stricto sensu em contabilidade das Gerações $\mathrm{Y}$ e $\mathrm{Z}$, considerando as características sociodemográficas?

Geralmente, pessoas das gerações $\mathrm{Y}$ e $\mathrm{Z}$ entram na pós-graduação stricto sensu no início da fase adulta e no início de sua trajetória profissional. Essa fase da vida é marcada por desafios, oportunidades e altas demandas por conquistas, a fim de se estabelecer no mundo do trabalho. Acredita-se que essas circunstâncias de vida incentivam os estudantes a priorizar valores de trabalho que se adequem às suas características e anseios. Assim, conhecer as diferenças de valores do trabalho das gerações $\mathrm{Y}$ e $\mathrm{Z}$, a partir de suas características sociodemográficas, é importante para compreender o comportamento no ambiente trabalho e suas escolhas, tendo em vista que o trabalho exerce influência na formação e no bem-estar do indivíduo (Cavazotte et al., 2012).

Em relação ao objeto de pesquisa, o estudo considerou o stricto sensu como análogo ao ambiente profissional para os estudantes, por ser um ambiente permeado de tarefas, metas e exigências que resultarão na recompensa do título. Além disso, estudos sobre os valores do trabalho no stricto sensu possibilita discussões entre os próprios indivíduos sobre suas escolhas, carreiras, motivações e interesses. Esses aspectos são de grande importância para promover a satisfação do indivíduo no ambiente de trabalho e, consequentemente, o desempenho da instituição em que trabalham.

A compreensão das características e valores das novas gerações abre a possibilidade de verificar se as preferências desses grupos são consistentes à formação que receberam durante a pós-graduação e se estão alinhadas às ofertas e demandas do mercado de trabalho. Aliado a isso, a partir da compreensão desses valores, os professores podem projetar práticas que atendam às expectativas das novas gerações e que promovam o entusiasmo do aluno por meio da exploração da carreira.

\section{Valores Relativos ao Trabalho e Gerações Y e Z}

Os valores relativos ao trabalho são concepções relacionadas aos objetivos desejados ou comportamentos no ambiente profissional. Tais objetivos são ordenados de acordo com sua importância, como princípios que orientam o indivíduo nas avaliações e escolhas no contexto do trabalho (Ros, Schwartz \& Surkiss, 1999). Roe e Ester (1999) expressam que os valores laborais são considerados fontes de motivação para o desempenho individual, e no aspecto social, exercem influência indireta, definem regras e objetivos compartilhados que orientam a ação coletiva.

Para Elizur (1984), os valores do trabalho estão relacionados a aspectos pessoais, considerando que os valores tidos como importantes no trabalho geram recompensa financeira ou exercício de funções proeminentes que afetam a vida pessoal do indivíduo. Por isso, esses valores devem ser analisados em conjunto para ajudar a explicar a sua importância no bem-estar da pessoa. Na mesma direção Ros et al., (1999, p. 54), declaram que os valores do trabalho são "expressões específicas dos valores pessoais no ambiente de trabalho". 
Estudos sobre os valores do trabalho buscam compreender o que é considerado significativamente importante para o indivíduo no ambiente de trabalho (Porto \& Tamayo, 2008). Entretanto, não só os indivíduos são detentores de valores, eles podem ser encontrados no âmbito coletivo, isto é, em pessoas que pertencem a um mesmo grupo, região geográfica, comunidade ou cultura (Roe \& Ester, 1999). Os valores são utilizados para explicar as mudanças na sociedade, analisar condutas e caracterizar grupos (Porto \& Tamayo, 2003).

Considerando que os indivíduos de uma mesma geração constituem um grupo que nasceram na mesma época e possuem características e experiências comuns, conjectura-se que, tais experiências podem afetar seus valores, crenças e comportamentos na vida e no ambiente de trabalho (Hajdu \& Sik, 2018). Estudos (Smola \& Sutton, 2002; Parry \& Urwin, 2011; Hajdu \& Sik, 2018) afirmaram que os valores de uma geração tendem a ser influenciados pelo período em que nasceram, pelos acontecimentos históricos e as experiências semelhantes (guerras, crises sociais, desastres naturais, mudanças políticas e tecnológicas) vivenciadas nos primeiros anos de vida, tornando-as semelhantes entre si e diferentes das outras gerações.

No ambiente de trabalho moderno, as diferenças entre as gerações remetem aos desafios organizacionais relacionados a liderança, comprometimento, relacionamento interpessoal, desempenho, entre outros (Comazzetto et al., 2016). Nas organizações, as atitudes, o desempenho no ambiente de trabalho e a forma como os funcionários se relacionam uns com outros são influenciados por seus valores, princípios e pela forma como pensam, os quais, muitas vezes, são comuns às pessoas de uma mesma geração. Grubb (2016) defende que para coordenar bem as pessoas é necessário compreendê-las. Por isso, qualquer pessoa que conduza um ambiente de trabalho multigeracional deve se atentar e privilegiar o entendimento dessas características e atitudes.

Mannheim (1993) considera que para estudar as gerações é importante compreender as vivências de situações históricas, e, acima de tudo, a forma como essas situações e experiências foram compreendidas e interiorizadas pelos indivíduos. A definição de gerações de Mannheim (1993) direciona o olhar sobre os comportamentos e as manifestações de determinados grupos em detrimento de tentar rotulá-los de forma pontual em relação ao aspecto temporal.

A Geração Y, em específico, cresceu em contato com a tecnologia e possui características mais individualistas. As pessoas dessa geração nasceram praticamente ao mesmo tempo que as evoluções tecnológicas e a globalização, e estes eventos influenciaram suas características, ideais e comportamentos (Santos Neto \& Franco, 2010; Comazzetto et al., 2016). Estudos reportam que pessoas da Geração Y, no contexto do trabalho, são inventivos, inovadores e confiantes; buscam o sucesso profissional imediato; esperam ser reconhecidos frequentemente; almejam por um trabalho que lhes deem realização além de remuneração. Por esses motivos, a Geração Y é mais provável de desistir de um trabalho que não lhe satisfaça (Santos Neto \& Franco, 2010; Kowske, Rasch, \& Wiley, 2010; Grubb, 2016; Comazzetto et al., 2016; Forbes, 2019).

Estudos conceituam a Geração Z como uma ampliação da geração Y (Santos Neto \& Franco, 2010; Ozkan \& Solmaz, 2015; Grubb, 2016). A Geração Z não conheceu o mundo sem internet, computadores ou telefones celulares. Seu comportamento e sua forma de pensar foram completamente influenciados pelo uso instantâneo da tecnologia (Faber, 2011). Para essa geração, o conceito de mundo é desprendido das limitações geográficas, e a tecnologia é utilizada como uma extensão de sua autoexpressão (Faber, 2011; Grubb, 2016). Além disso, os jovens dessa geração são caracterizados por terem alta facilidade com mídias sociais, valores voltados para o futuro, além de serem dinâmicos, adeptos a mudanças e multitarefas (Ozkan \& Solmaz, 2015; Revista Exame, 2018). 
A relevância em compreender as diferenças entre os comportamentos e as crenças geracionais desencadeou diferentes estudos. Cennamo e Gardner (2008) analisaram as diferenças entre a Geração Baby Boomers, Geração X e Geração Y em valores do trabalho, satisfação, comprometimento e intenções de sair. Participaram da pesquisa 504 funcionários de uma organização e os resultados desmontaram que a geração mais jovem (Y) dá mais importância a valores como status, liberdade e envolvimento social. Os Baby Boomers atribuem maior importância a valores de organização e valores extrínsecos em relação às Gerações X e Y.

Twenge et al. (2010) também analisaram as diferenças entre a geração Baby Boomers, Geração X e Geração Y e seus valores relativos ao trabalho. A pesquisa foi realizada com 16.507 estudantes do ensino médio dos EUA, nos anos de 1976, 1991 e 2006, de forma que contemplasse as três gerações. Os resultados evidenciaram que os valores de lazer aumentaram significativamente ao longo dos anos comparando a Gerações Baby Boomers e Y; a centralidade do trabalho decresceu e os valores relacionados a status e dinheiro atingiram níveis elevados para a Geração X e mais ainda para a Geração Y, quando comparados à Geração Baby Boomers.

Em contrapartida, os resultados do estudo de Kowske et al., (2010) evidenciaram que as Gerações Baby Boomers, X e Y são mais semelhantes do que diferentes, quando se trata de valores relativos ao trabalho. Também Maloni, Hiatt e Campbell (2019), ao analisarem os valores desejados por estudantes das Gerações Y e Z e a compreensão desses valores por parte dos docentes e recrutadores da área de negócios, descobriram que essas/ gerações apresentam amplas semelhanças quanto aos seus valores do trabalho, principalmente quando se referem a retorno financeiro e estabilidade no trabalho.

Os resultados de estudos que abordam os valores geracionais não indicam direções consistentes reforçando a necessidade de pesquisas empíricas sobre o tema. Nesse contexto, Mannheim (1993) sugere analisar o motivo das ações dos indivíduos que compõe uma mesma geração, o que implica na observação sob o ponto de vista macro, que corresponde, portanto, à conjuntura histórica, política e social, e sob o ponto de vista micro, que corresponde ao conhecimento adquirido pelo indivíduo ao longo de seu desenvolvimento (Weller, 2010). Características pessoais como idade, gênero, etnia, ocupação profissional e o contexto social tendem a diferençar os valores do trabalho entre os indivíduos (MOW, 1987; Parry \& Urwin, 2011; Cavazotte et al., 2012).

Cavazotte, et al., (2012) afirmam que as características sociodemográficas afetam os valores relativos ao trabalho, bem como o contexto histórico e social em que o indivíduo se desenvolveu. Para Schwartz (2006), tudo o que afeta as circunstâncias de vida as quais os indivíduos devem se adaptar pode influenciar os valores relativos ao trabalho. A idade, a educação, o gênero e as diversas características do indivíduo definem amplamente as circunstâncias de vida que estão expostos e tendem a afetar os valores do trabalho. Por isso, assume-se como hipótese desse estudo que: há diferenças de valores relativos ao trabalho de estudantes do stricto sensu em contabilidade das Gerações Y e Z quando se considera as características sociodemográficas dos indivíduos.

\section{Metodologia}

O estudo caracteriza-se como descritivo com abordagem quantitativa. A população foi composta pelos 1.696 estudantes do stricto sensu em Contabilidade e amostra final de 337 estudantes que em 2019 estavam regularmente matriculados em um dos programas de pós-graduação vinculados à área contábil. A coleta de dados deu-se por meio do instrumento eletrônico aplicado on-line utilizando o software SurveyMonkey ${ }^{\circledast}$ durante os meses de outubro e novembro de 2019. 
O instrumento utilizado na pesquisa foi constituído de duas seções. A primeira teve o propósito de identificar os valores relativos ao trabalho por meio da Escala Revisada de Valores Relativos ao Trabalho (EVT-R) desenvolvida e autorizada para uso por Porto e Pilati (2010). A EVT-R é composta de34 valores relativos ao trabalho. Os respondentes deveriam indicar o quão importante é cada um utilizando a escala que varia de "1 - nada importante a 5 - extremamente importante". Na Figura 1, apresentam-se os itens que compuseram a EVT-R.

\begin{tabular}{|c|c|}
\hline ID & É importante para mim... \\
\hline Q1 & Ajudar os outros \\
\hline Q2 & Colaborar para o desenvolvimento da sociedade \\
\hline Q3 & Combater injustiças sociais \\
\hline Q4 & Demonstrar minhas competências \\
\hline Q5 & Obter estabilidade financeira \\
\hline Q6 & Ganhar dinheiro \\
\hline Q7 & Obedecer às normas do trabalho \\
\hline Q8 & Poder me sustentar financeiramente \\
\hline Q9 & Respeitar a hierarquia \\
\hline Q10 & Ser admirado pelo meu trabalho \\
\hline Q11 & Ser bem-sucedido na minha profissão \\
\hline Q12 & Ser independente financeiramente \\
\hline Q13 & Ser reconhecido pelo resultado satisfatório do meu trabalho \\
\hline Q14 & Ser respeitado pelas minhas competências no trabalho. \\
\hline Q15 & Ser útil para a sociedade \\
\hline Q16 & Supervisionar outras pessoas \\
\hline Q17 & Ter autonomia na realização das minhas tarefas \\
\hline Q18 & Ter compromisso social \\
\hline Q19 & Ter desafios constantes \\
\hline Q20 & Ter fama \\
\hline Q21 & Ter liberdade para decidir a forma de realização do meu trabalho \\
\hline Q22 & Ter melhores condições de vida \\
\hline Q23 & Ter prestígio \\
\hline Q24 & Ter um trabalho arriscado \\
\hline Q25 & Ter um ambiente de trabalho com hierarquia clara \\
\hline Q26 & Ter um trabalho criativo \\
\hline Q27 & Ter um trabalho inovador \\
\hline Q28 & Ter um trabalho organizado \\
\hline Q29 & Ter um trabalho que me permita conhecer lugares novos \\
\hline Q30 & Ter um trabalho que me permita conhecer pessoas novas \\
\hline Q31 & Ter um trabalho que me permita expressar meus conhecimentos \\
\hline Q32 & Ter um trabalho que requer originalidade \\
\hline Q33 & Ter uma profissão reconhecida socialmente \\
\hline Q34 & Competir com colegas de trabalho para alcançar as minhas metas profissionais \\
\hline
\end{tabular}

Figura 1. Escala Revisada de Valor Relativo ao Trabalho (EVT-R)

Fonte: Porto e Pilati (2010). 
A segunda seção teve como objetivo coletar dados sociodemográficos. Para distinguir o período de corte das Gerações Y e Z, foi utilizado o trabalho de Santos Neto e Franco (2010), em virtude de ser um estudo essencialmente brasileiro e considerar elementos históricos, políticos e sociais na constituição das gerações. Classificaram-se como da Geração Y as pessoas nascidas entre 1979-1992 e da Z aquelas nascidas a partir de 1993. Para garantir a integridade dos participantes bem como contribuir com pesquisas dentro dos padrões éticos, o presente estudo foi submetido e aprovado no Comitê de Ética em Pesquisa CEP/SD, registrado sob o número: 18268819.4.0000.0102.

\section{Descrição e Análise dos Dados}

\subsection{Resultados Descritivos}

A amostra final foi composta de 337 estudantes. Desses, 246 são da Geração Y, 91 da Geração Z e a maioria são do gênero feminino $(53,1 \%)$. Quanto à etnia, há predominância de estudantes que se autodeclaram da etnia branca (61,7\%), seguido da parda (29,4\%), preta (5,9\%), amarela (2,7\%) e indígena $(0,3 \%)$. Na Tabela 1 , apresentam-se as informações a respeito do perfil dos participantes que compuseram a amostra final do estudo.

Tabela 1

Perfil dos respondentes - características pessoais

\begin{tabular}{|c|c|c|c|c|c|}
\hline Geração & $\mathbf{F}$ & $\%$ & Experiência docente & $\mathbf{F}$ & $\%$ \\
\hline Geração Y (1979-1992) & 246 & $73 \%$ & Sim & 185 & $55 \%$ \\
\hline Geração Z (a partir de 1993) & 91 & $27 \%$ & Não & 152 & $45 \%$ \\
\hline Gênero & $\mathbf{F}$ & $\%$ & Experiência profissional & $\mathbf{F}$ & $\%$ \\
\hline Feminino & 179 & $53,1 \%$ & Sim & 303 & $90 \%$ \\
\hline Masculino & 156 & $46,3 \%$ & Não & 34 & $10 \%$ \\
\hline Agênero ou Não-binário & 1 & $0,3 \%$ & Fase da pós-graduação & $\mathbf{F}$ & $\%$ \\
\hline Prefiro não responder & 1 & $0,3 \%$ & Cursando os créditos & 90 & $27 \%$ \\
\hline Etnia & $\mathbf{F}$ & $\%$ & Em fase de qualificação & 72 & $21 \%$ \\
\hline Amarela & 9 & $2,7 \%$ & Em fase de pós qualificação & 42 & $12 \%$ \\
\hline Branca & 208 & $61,7 \%$ & Próximo a minha defesa final & 56 & $17 \%$ \\
\hline Indígena & 1 & $0,3 \%$ & Já defendi minha dissertação ou tese & 77 & $23 \%$ \\
\hline Parda & 99 & $29,4 \%$ & Auxílio Financeiro na pós-graduação & $\mathbf{F}$ & $\%$ \\
\hline \multirow[t]{3}{*}{ Preta } & 20 & $5,9 \%$ & Sim. Durante todo o curso & 104 & $31 \%$ \\
\hline & & & Sim, somente em uma parte do curso & 57 & $17 \%$ \\
\hline & & & Não, nunca recebi auxílio financeiro & 176 & $52 \%$ \\
\hline
\end{tabular}

Nota: $\mathrm{F}=$ frequência; $\%$ = percentual.

Fonte: dados da pesquisa (2021). 
A maior parte dos respondentes afirmaram ter alguma experiência com a docência (55\%) e experiência profissional (90\%). Em relação à pós-graduação, 27\% estão cursando os créditos, enquanto $23 \%$ já defenderam a dissertação ou tese. Complementarmente, a maioria dos respondentes (52\%) declararam nunca ter recebido nenhum tipo de bolsa. Na Tabela 2, apresenta-se o perfil dos respondentes considerando o contexto familiar.

Tabela 2

\section{Perfil dos respondentes - contexto familiar}

\begin{tabular}{lcclcc}
\hline Escolaridade da figura Materna & $\mathbf{F}$ & \% & Escolaridade da figura Paterna & F & $\%$ \\
\hline Não concluiu o Ensino Fundamental & 64 & $19 \%$ & Não concluiu o Ensino Fundamental & 77 & $23 \%$ \\
Concluiu o Ensino Fundamental & 31 & $9,2 \%$ & Concluiu o Ensino Fundamental & 37 & $11 \%$ \\
Não concluiu o Ensino Médio & 21 & $6,2 \%$ & Não concluiu o Ensino Médio & 27 & $8 \%$ \\
Ensino Médio & 117 & $35 \%$ & Ensino Médio & 105 & $31 \%$ \\
Ensino Superior - Graduação & 52 & $15,4 \%$ & Ensino Superior - Graduação & 51 & $15 \%$ \\
Ensino Superior - Especialização & 41 & $12,2 \%$ & Ensino Superior - Especialização & 27 & $8 \%$ \\
Ensino Superior - Stricto Sensu & 10 & $3 \%$ & Ensino Superior - Stricto Sensu & 11 & $3 \%$ \\
Desconheço & 1 & $0 \%$ & Desconheço & 2 & $1 \%$ \\
\hline Responsabilidade financeira em casa & $\mathbf{F}$ & $\%$ & Horas conectado à internet & $\mathrm{F}$ & $\%$ \\
\hline Sou o único responsável & 77 & $23 \%$ & Menos de 2 horas & 17 & $5 \%$ \\
Principal responsável e recebo ajuda & 61 & $18 \%$ & De 2 a 6 horas & 113 & $34 \%$ \\
Divido igualmente com outra pessoa & 96 & $28 \%$ & De 6 a 10 horas & 109 & $32 \%$ \\
Contribuo com uma pequena parte & 74 & $22 \%$ & De 10 a 16 horas & 88 & $26 \%$ \\
Nenhuma responsabilidade financeira & 29 & $9 \%$ & 24 horas por dia & 10 & $3 \%$ \\
\hline
\end{tabular}

Nota: $F$ = frequência; $\%$ = percentual.

Fonte: dados da pesquisa (2021).

No que se refere ao nível de escolaridade, a figura materna da maioria dos respondentes concluiu o ensino médio (35\%). O mesmo cenário é observado para a figura paterna da maioria dos respondentes, uma vez que $31 \%$ tem como maior nível de escolaridade o ensino médio. Em relação à responsabilidade financeira em casa, a maioria dos respondentes (28\%) divide igualmente as responsabilidades com outra pessoa, enquanto (9\%) afirmaram não ter nenhuma responsabilidade financeira em casa. Por fim, apesar das Gerações Y e Z serem consideradas hiperconectadas (Grubb, 2016), apenas 3\% dos participantes desta pesquisa consideram que permanecem $24 \mathrm{~h}$ por dia conectados. 


\subsection{Análise dos Valores do Trabalho Considerando Variáveis Sociodemográficas}

Inicialmente, a Análise Fatorial Exploratória (AFE) foi empregada com o intuito de verificar a formação dos fatores provenientes da EVT-R. Para utilizar a AFE, é necessário verificar alguns critérios, quais sejam: comunalidades (acima de 0,50); KMO (acima de 0,70), teste de Esfericidade de Bartlett ( $p$-value $<0,050$ ), Medida de Adequação da Amostra-MAS (acima de 0,7) e cargas fatorais (acima de 0,4 ) (Hair, Black, Babin, Anderson e Thatam, 2009). Ao analisar as comunalidades, verificou-se que cinco variáveis (Q1, Q24, Q28, Q33 e Q34) apresentaram valores inferiores ao parâmetro de 0,5. Em razão disso, tais variáveis foram excluídas por não atingirem o valor mínimo aceitável (Hair et al., 2009). Os resultados das análises evidenciaram que o KMO foi satisfatório $(0,886)$, o teste de esfericidade de Bartlett significativo $(0,000)$ e o MAS admirável (entre 0,824 e 0,946$)$.

Tais resultados indicam adequação do uso da AFE para a amostra utilizada neste estudo. Outro critério aplicado na análise concerne na porcentagem da variância explicada que deve ser próximo a $60 \%$ (Hair et al.,2009). A AFE resultou na formação de sete fatores, os quais apresentaram itens com carga superior a 0,4 , que juntos explicam $68,81 \%$ da variância total explicada. O método utilizado para a extração dos fatores foi o de componentes principais com rotação Varimax. Posteriormente, foi verificada a confiabilidade e a consistência interna da EVT-R por meio do Alfa de Cronbach, o qual apresentou valores satisfatórios, acima de 0,7, em todos os fatores (Hair et al., 2009). Na Figura 2, apresenta-se a composição dos fatores, a nomenclatura atribuída a cada um deles, bem como os itens que os compõem.

\begin{tabular}{lll}
\hline Fatores & Descrição & Itens \\
\hline Fator 1 - Estimulação & $\begin{array}{l}\text { Valores relacionados a inovação, desafios na vida e } \\
\text { mudanças desejadas. }\end{array}$ & $\begin{array}{l}\text { Q26; Q27; Q32; Q30; } \\
\text { Q29; Q31; Q19 }\end{array}$ \\
\hline Fator 2 - Realização & $\begin{array}{l}\text { Valores relacionados a sucesso profissional, satisfação } \\
\text { pelo trabalho exercido e exibição de competências. }\end{array}$ & Q13; Q10; Q11; Q14; Q4 \\
\hline Fator 3 - Segurança & $\begin{array}{l}\text { Valores relacionados a retorno financeiro e estabilidade } \\
\text { no trabalho e independência financeira. }\end{array}$ & Q8; Q12; Q5; Q6; Q22 \\
\hline $\begin{array}{l}\text { Fator } 4 \text { - Universalismo e } \\
\text { Benevolência }\end{array}$ & $\begin{array}{l}\text { Valores que exprimem atenção aos aspectos sociais e } \\
\text { preocupação com outro. }\end{array}$ & Q2; Q3; Q18; Q15 \\
\hline Fator 5 - Poder & $\begin{array}{l}\text { Valores relacionados a status social, prestígio, } \\
\text { superioridade e influência sobre as pessoas. }\end{array}$ & Q20; Q23; Q16 \\
\hline Fator 6 - Conformidade & $\begin{array}{l}\text { Valores referentes à hierarquia, à aceitação de normas, } \\
\text { rotinas de trabalho e disciplina. }\end{array}$ & Q9; Q7; Q25 \\
\hline Fator 7 - Autodeterminação & $\begin{array}{l}\text { Valores relacionados a iniciativa, liberdade de } \\
\text { pensamento e independência. }\end{array}$ & Q17; Q21 \\
\hline
\end{tabular}

Figura 2. Composição e descrição dos fatores

Fonte: dados da pesquisa (2021).

Após verificar a formação dos fatores resultantes dos valores elencados na EVT-R, para analisar se há diferenças de valores do trabalho entre os respondentes, foram realizados testes de diferenças entre os grupos. Para isso, inicialmente foi necessário realizar o teste de normalidade, a fim de identificar qual técnica estatística seria mais adequada para a amostra estudada. A normalidade dos fatores formados pela EVT-R foi verificada por meio do teste Kolmogorov-Smirnov. Os resultados do teste indicaram que três fatores se mostraram significativos ( $p$ value $<0,05)$. Isso quer dizer que esses três fatores não apresentam distribuição normal (Field, 2009). Apesar de apenas três dos sete fatores apresentarem distribuição não normal, optou-se por utilizar o teste não paramétrico de Kruskal-Wallis (K-W), tendo em vista que o conjunto total dos dados apresenta natureza categórica e que houve a violação das suposições dos testes paramétricos em três fatores. 
O teste de Kruskal-Wallis (K-W) foi utilizado com o intuito de verificar se existem diferenças entre os valores do trabalho dos respondentes e a variáveis sociodemográficas. Em seguida, como post-hoc, foi aplicado o teste Pairwise Method para comparar todos os pares possíveis dentro de cada grupo, a fim de controlar erros do Tipo I. Para isso, o $p$-value é dividido pelo número de comparações dentro de cada grupo, certificando que o erro do Tipo I seja menor que 0,05 (Field, 2009).

Cada fator formado pela EVT-R foi testado separadamente com as variáveis sociodemográficas. Os testes não paramétricos para os Fatores 1 "Estimulação", 2 "Realização", 3 "Segurança” e 4 "Universalismo e Benevolência” são apresentados na Tabela 3.

Tabela 3

Teste não paramétricos para os Fatores 1, 2, 3 e 4

\begin{tabular}{|c|c|c|c|c|c|}
\hline \multirow{2}{*}{ Teste NP } & \multirow{2}{*}{ Variáveis Sociodemográficas } & \multicolumn{4}{|c|}{ Fatores } \\
\hline & & $\mathrm{E}$ & R & $S$ & UB \\
\hline K-W & Gênero & 0,452 & 0,073 & $0,001 *$ & 0,931 \\
\hline \multicolumn{6}{|l|}{1 de 6 pares } \\
\hline Pairwise & (Feminino) e (Masculino) & & & $0,001 *$ & \\
\hline \multirow{2}{*}{ MR } & feminino & & & 187,44 & \\
\hline & masculino & & & 148,06 & \\
\hline K-W & Etnia & 0,398 & 0,477 & 0,624 & 0,723 \\
\hline K-W & Nível de escolaridade da figura Materna & 0,468 & 0,375 & 0,727 & 0,531 \\
\hline K-W & Nível de escolaridade da figura Paterna & 0,594 & 0,128 & 0,781 & 0,398 \\
\hline K-W & Responsabilidade financeira em casa & $0,030 *$ & 0,424 & 0,679 & 0,785 \\
\hline \multicolumn{6}{|l|}{1 de 10 pares } \\
\hline Pairwise & (contribuo c/ uma parte) e (principal respon.) & $0,037 * *$ & & & \\
\hline \multirow{2}{*}{$M R$} & contribuo apenas com uma parte & 147,34 & & & \\
\hline & principal responsável, mas recebo ajuda & 196,26 & & & \\
\hline K-W & Auxílio Financeiro durante a pós-graduação & 0,512 & 0,482 & 0,101 & 0,421 \\
\hline K-W & Horas conectado à internet & 0,346 & 0,844 & 0,288 & 0,836 \\
\hline K-W & Experiência docente & $0,002^{*}$ & 0,882 & 0,279 & 0,289 \\
\hline \multicolumn{6}{|l|}{1 par } \\
\hline Pairwise & (sim) e (não) & $0,002^{*}$ & & & \\
\hline \multirow{2}{*}{$M R$} & sim & 183,56 & & & \\
\hline & não & 151,28 & & & \\
\hline K-W & Experiência profissional & 0,757 & 0,824 & 0,843 & 0,187 \\
\hline K-W & Fase da pós-graduação & 0,567 & 0,282 & 0,479 & 0,06 \\
\hline
\end{tabular}

Nota: $\left(^{*}\right)$ significância a 5\%;(**) significância ajustada; MR= Mean Rank; E = Estimulação; R = Realização; S = Segurança; UB $=$ Universalismo e Benevolência.

Fonte: dados da pesquisa (2021). 
Os resultados do teste de $\mathrm{K}-\mathrm{W}$ apontaram que a importância atribuída aos valores que compõem o Fator "Estimulação" é significativamente diferente conforme a "responsabilidade financeira em casa" e a "experiência docente" dos respondentes das Gerações Y e Z. A variável "responsabilidade financeira em casa" indicou haver diferenças significantes entre o grupo "Contibuo apenas com uma pequena parte" ( $M R=147,34)$ e o grupo "Principal responsável, mas recebo ajuda" ( $M R=196,26)$. Conforme indicado pelo Mean Rank, pode-se inferir que os respondentes que declararam ser o principal responsável pelas despesas de casa, atribuem maior importância aos valores do trabalho relacionados à inovação, desafios na vida e mudanças desejadas, os quais constituem o Fator "Estimulação".

Em relação à variável "experiência docente", os respondentes que possuem experiência docente $(M R=$ 183,56) atribuem maior importância aos valores associados à "Estimulação" no ambiente de trabalho quando comparados aos respondentes que não possuem experiência docente $(M R=151,28)$. Na mesma direção, Schwartz (2006) exprime que experiências educacionais promovem abertura intelectual e flexibilidade; tais experiências ampliam horizontes e direcionam o indivíduo a priorizar valores de estimulação.

Além disso, os resultados indicaram diferenças para a importância atribuída aos valores que compõem o Fator "Segurança" no que diz respeito ao gênero. A comparação entre os pares apresentou diferenças significativas entre o gênero feminino $(M R=187,44)$ e o masculino $(M R=148,06)$. Isso quer dizer que os respondentes do gênero feminino atribuem maior importância aos valores associados a retorno financeiro e estabilidade no trabalho, os quais compõem o Fator "Segurança", quando comparados aos respondentes do gênero masculino.

Esse resultado é oposto aos achados de Potrich, Vieira, Estivalete e Andrade (2015), que não indicaram diferenças significativas entre gênero e o Fator "Segurança". Por outro lado, os resultados do estudo Estivalete, Lõbler, Andrade e Visentini (2011) e de Silva, Mendonça e Zanini (2010) evidenciaram que as mulheres tendem a priorizar valores associados a estabilidade financeira, independência financeira e autossustento, os quais se referem ao Fator "Segurança”. Esses resultados podem estar atrelados ao crescente papel da mulher na estrutura familiar, como provedora ou parte importante na composição da renda familiar. 
O teste de K-W não apresentou diferenças significativas para o Fatores "Realização" e "Universalismo e Benevolência”. Na Tabela 4, apresentam-se os testes não paramétricos para os Fatores 5 "Poder", 6 "Conformidade" e 7 "Autodeterminação".

Tabela 4

Teste não paramétricos para os Fatores 5, 6 e 7

\begin{tabular}{|c|c|c|c|c|}
\hline \multirow{2}{*}{ Teste NP } & \multirow{2}{*}{ Variáveis Sociodemográficas } & \multicolumn{3}{|c|}{ Fatores } \\
\hline & & $\mathbf{P}$ & C & A \\
\hline K-W & Gênero & 0,622 & 0,503 & 0,133 \\
\hline K-W & Etnia & 0,359 & 0,392 & 0,923 \\
\hline K-W & Nível de escolaridade da figura Materna & 0,315 & $0,004^{*}$ & 0,902 \\
\hline \multicolumn{5}{|l|}{1 de 28 pares } \\
\hline Pairwise & (concluiu o E.S.- Especialização) e (concluiu o E.M.) & & $0,018 * *$ & \\
\hline \multirow{2}{*}{$M R$} & concluiu o Ensino Superior - Especialização & & 131,37 & \\
\hline & concluiu o Ensino Médio & & 191,83 & \\
\hline K-W & Nível de escolaridade da figura Paterna & 0,729 & 0,377 & 0,847 \\
\hline K-W & Responsabilidade financeira em casa & 0,358 & 0,714 & 0,239 \\
\hline K-W & Auxílio Financeiro durante a pós-graduação & 0,877 & $0,001 *$ & 0,79 \\
\hline \multicolumn{5}{|l|}{2 de 3 pares } \\
\hline Pairwise & (sim. Em parte do curso) e (Não. Nunca recebi) & & $0,012 * *$ & \\
\hline \multirow{2}{*}{$M R$} & sim. Somente em uma parte do curso & & 144,74 & \\
\hline & não. Nunca recebi nenhum tipo de bolsa & & 187,55 & \\
\hline Pairwise & (sim. Durante todo o curso) e (Não. Nunca recebi) & & $0,007 * *$ & \\
\hline \multirow{2}{*}{ MR } & sim. Durante todo o curso & & 150,91 & \\
\hline & não. Nunca recebi nenhum tipo de bolsa & & 187,55 & \\
\hline K-W & Horas conectado à internet & $0,045 *$ & 0,879 & 0,433 \\
\hline K-W & Experiência docente & 0,961 & 0,332 & $0,036 *$ \\
\hline \multicolumn{5}{|l|}{1 par } \\
\hline Pairwise & (sim) e (não) & & & $0,036 *$ \\
\hline \multirow{2}{*}{$M R$} & $\operatorname{sim}$ & & & 179,07 \\
\hline & não & & & 156,74 \\
\hline K-W & Experiência profissional & 0,41 & 0,534 & 0,713 \\
\hline K-W & Fase da pós-graduação & 0,511 & $0,019 *$ & 0,071 \\
\hline \multicolumn{5}{|l|}{1 de 10 pares } \\
\hline Pairwise & (em fase de qualificação) e (próximo a minha defesa) & & $0,009 * *$ & \\
\hline \multirow{2}{*}{$M R$} & em fase de qualificação & & 146,32 & \\
\hline & próximo a minha defesa final & & 203,84 & \\
\hline
\end{tabular}

Nota: ${ }^{*}$ ) significância a 5\%; (**) significância ajustada; MR= Mean Rank; $\mathrm{P}=$ Poder; $\mathrm{C}=$ Conformidade; $\mathrm{A}$ = Autodeterminação

Fonte: dados da pesquisa (2021) 
Os resultados do teste $\mathrm{K}-\mathrm{W}$ evidenciaram diferenças significativas entre a importâcia atribuída aos valores que compõem o Fator "Poder" e as "horas conectado à internet". Contudo, após a comparação entre os pares dessa variável, verificou-se não haver diferenças significativas entre os grupos. Isso ocorre porque a comparação entre pares implica o ajuste do $p$-value conforme a quantidade de comparações feitas dentro do grupo.

Nota-se que houve diferenças significativas na importância atribuída aos valores que constituem o Fator "Conformidade", segundo o "nível de escolaridade da figura materna", "Auxílio financeiro durante a pós-graduação" e a "fase da pós-graduação". As diferenças significativas da variável "nível de escolaridade da Figura materna" ocorreram entre o grupo que "Concluiu o ensino superior - Especialização" $(M R=131,37)$ e o grupo que "Concluiu o ensino médio" $(\mathrm{MR}=191,83)$. Esse resultado demonstra que os respondentes das Gerações Y e Z, cuja figura materna concluiu somente o ensino médio, atribuem maior importância aos valores relacionados à hierarquia e normas de trabalho os quais compõem o fator Conformidade.

Ambiel, Ferraz, Pereira, Simões e Silva (2019) destacaram que o apoio dos pais em relação ao aspecto profissional dos filhos é diferenciado em função dos níveis de escolaridade e profissional dos pais. Assim, pais com níveis de escolaridade mais altos tendem a ajudar os filhos na escolha da formação, muitas vezes com apoio especializado de psicólogos para auxiliar na compreensão da carreira e perspectivas profissionais dos filhos. No entanto, pais com níveis de escolaridade mais baixos, parecem não estimular os filhos nas escolhas profissionais e nas atitudes perante o trabalho, muitas vezes por não entenderem $o$ suficiente sobre o assunto (Ambiel et al., 2019).

Para a variável "Auxílio financeiro durante a pós-graduação", houve diferenças significativas entre o grupo que recebeu auxílio financeiro durante uma parte do curso $(M R=144,74)$ e o grupo que nunca recebeu nenhum tipo de bolsa $(M R=187,55)$. Os respondentes que nunca receberam nenhum tipo de bolsa atribuem maior importância aos valores associados à hierarquia e normas de trabalho os quais constituem o fator "Conformidade", quando comparados aos respondentes que receberam auxílio financeiro durante uma parte do curso.

Ainda em relação à variável "Auxílio financeiro durante a pós-graduação", verificou-se diferenças significativas entre o grupo que recebeu bolsa durante todo o curso $(M R=150,91)$ e o grupo que nunca recebeu nenhum tipo de bolsa $(\mathrm{MR}=187,55)$. Igualmente, os respondentes que nunca receberam nenhum tipo de bolsa durante a pós-graduação atribuem maior importância aos valores que constituem o Fator "Conformidade". Referente à variável "fase da pós-graduação", notam-se diferenças significativas entre o grupo que está "em fase de qualificação" ( $\mathrm{MR}=146,32)$ e o grupo que está "próximo à defesa final” ( $\mathrm{MR}=$ $203,84)$. Pode-se inferir que os respondentes que estão próximos à defesa final atribuem maior importância aos valores relacionados à hierarquia e normas de trabalho, quando comprados aos respondentes que estão em fase de qualificação.

Também foram constatadas diferenças significativas entre a importância atribuída aos valores que compõem o Fator "Autodeterminação" e a variável " experiência docente". Nessa variável, foram verificadas diferenças significativas entre o grupo com experiência docente $(M R=179,07)$ e o grupo sem experiência docente $(M R=156,74)$. Esse resultado indica que os respondentes com experiência docente atribuem maior importância aos valores relacionados à iniciativa, liberdade de pensamento e independência, quando comparados aos respondentes sem experiência docente. Também Schwartz (2006) afirma que a experiência educacional suscita valores de autodeterminação, isto porque as competências adquiridas para lidar com pessoas por meio da educação promovem valores relacionados à disposição e amplitude de perspectivas. 
Dessa forma, os resultados confirmaram a hipótese que os valores relativos ao trabalho se diferem conforme as características sociodemográficas do indivíduo. Neste estudo, os valores se diferenciaram de acordo com gênero, experiência docente, responsabilidade financeira em casa, nível de escolaridade da figura materna, auxílio financeiro durante a pós-graduação e fase da pós-graduação. Todas essas características impactam algum dos valores relativos do trabalho dos respondentes. Apesar do estudo presentar uma hipótese intuitiva em relação aos estudantes do stricto sensu em Contabilidade das Gerações Y e Z, os resultados corroboram os estudos sobre os valores do trabalho, que já indicavam haver as diferenças de valores e as circunstâncias de vida do indivíduo (MOW, 1987; Schwartz, 2006; Parry \& Urwin, 2011; Cavazotte et al., 2012).

Ainda que as Gerações Y e Z tenham amplas semelhanças e comportamentos compartilhados (Kowske et al.,2010; Ozkan \& Solmaz, 2015; Maloni et al., 2019), é importante avaliar continuamente as diferenças de valores do trabalho dessas gerações mais jovens. Isso porque compreender os valores do trabalho em constante mudanças pode ajudar professores, programas de pós-graduação e gestores a atenderem melhor os anseios e as preferências dessas gerações.

\section{Conclusões}

Os valores do trabalho ajudam a compreender o que é importante para as pessoas e o que as motiva. Além disso, por meio dos valores é possível verificar mudanças de comportamento e diferenças entre grupos no ambiente de trabalho. Assim, objetivo deste trabalho consistiu em verificar as diferenças de valores relativos ao trabalho de estudantes do stricto sensu em Contabilidade das Gerações Y e Z, considerando as características sociodemográficas.

Estudantes que se identificam com o gênero feminino atribuíram maior importância aos valores relacionados à estabilidade no trabalho e independência financeira, quando comparados aos respondentes que se identificam com o gênero masculino. Esse resultado, em particular, corrobora outros encontrados na literatura (Estivalete et al.,2011; Silva et al., 2010), os quais evidenciaram que as mulheres tendem a priorizar a estabilidade financeira e o autossustento. Esses achados podem estar atrelados à participação ativa da mulher na composição da renda familiar e ao espaço que elas vêm conquistando no mercado de trabalho (Estivalete et al., 2011). Igualmente, foram encontradas diferenças significativas entre os grupos que compuseram as variáveis "responsabilidade financeira em casa", "experiência docente", "nível de escolaridade da figura materna" e "auxílio financeiro durante a pós-graduação".

Os resultados demostraram que as variáveis sociodemográficas causam variações significativas nos valores do trabalho das Gerações Y e Z. Embora as Gerações Y e Z apresentem características semelhantes (Ozkan \& Solmaz, 2015; Maloni et al., 2019), pode-se constatar que existem diferenças quando se observam características mais específicas. A constatação dessas diferenças revela o quanto o ambiente de trabalho é diversificado e como as vivências de vida de cada um impactam seus valores trabalho e, consequentemente, o ambiente de trabalho

Os resultados deste estudo mostram a relevância de pesquisas acadêmicas sobre a temática laboral. Entender as características e valores do trabalho das Gerações Y e Z é o primeiro passo para compreender as diversas necessidades dessas gerações em relação ao trabalho. As características do indivíduo determinam as oportunidades e as restrições que as pessoas enfrentam em relação ao trabalho e seus recursos para lidar com elas. Por fim, os valores do trabalho podem influenciar os desenvolvimentos de habilidades, a escolha de oportunidades e até mesmo as mudanças de ambientes de trabalho depois de um tempo.

Uma das limitações da pesquisa refere-se à escassez de trabalhos que discutem a temática geracional juntamente com os valores do trabalho, ou que discutem os valores do trabalho na área de Contabilidade, o que dificultou o as discussões e inferências nos resultados. Adicionalmente, pesquisas futuras poderiam investigar os valores relativos ao trabalho e sua interação com características mais específicas da pessoa, como os traços de personalidade. Estes também são considerados variáveis que podem influenciar escolhas e atitudes dos indivíduos, na medida em que predizem os valores do trabalho. 


\section{Referências}

Almeida, J. E. F. (2020). Revolução tecnológica no mundo dos negócios e algumas oportunidades e desafios na área contábil. Revista De Contabilidade E Organizações, 14, e165516. Doi: https://doi. org/10.11606/issn.1982-6486.rco.2020.165516

Almeida, G. T. de, \& Silva, C. A. R. da. (2018). Os desafios dos gestores na carreira contábil: A perspectiva das gerações Baby Boomer, X, Y e Z. Enfoque: Reflexão Contábil, 37(4), 105-120. Doi: https://doi. org/10.4025/enfoque.v37i4.37052

Ambiel, R. A., Ferraz, A., Pereira, E., Simões, N., \& Silva, J. (2019). Predição da definição da escolha vocacional a partir de variáveis familiares. Avances en psicología latinoamericana, 37(1), 89-101. Doi: https://dx.doi.org/10.12804/revistas.urosario.edu.co/apl/a.6193

Blanch, J.M. (2007). Psicología Social del Trabajo. En M. Aguilar y A. Reid (Coords.). Tratado de Psicología Social. Perspectivas Socioculturales (pp. 210-238). México; Barcelona: Anthropos; UAM.

Berings, D., De Fruyt, F., \& Bouwen, R. (2004). Work values and personality traits as predictors of enterprising and social vocational interests. Personality and Individual Differences, 36(2), 349-364.

Cammarosano, M., Santos, F. C. A., \& Rojas, F. A. (2014). Valores relativos ao trabalho de pesquisadores em uma organização brasileira. RAE-Revista de Administração de Empresas, 54(4), 445-457. Doi: https://doi.org/10.1590/S0034-759020140409

Cavazotte, F. D. S. C. N., Lemos, A. H. C., \& Viana, M. D. A., (2012). Novas gerações no mercado de trabalho: expectativas renovadas ou antigos ideais? CADERNOS EBAPE. BR, 10(1), 162-180. Doi: https://doi.org/10.1590/S1679-39512012000100011

Cennamo, L., \& Gardner, D. (2008). Generational differences in work values, outcomes and personorganisation values fit. Journal of Managerial Psychology, 23(8), 891-906. Doi: https://doi. org/10.1108/02683940810904385

Comazzetto, L. R., Perrone, C. M., Vasconcellos, S. J. L., \& Gonçalves, J. (2016). A geração Y no mercado de trabalho: um estudo comparativo entre gerações. Psicologia Ciência e Profissão, 36(1), 145-157. Doi: https://doi.org/10.1590/1982-3703001352014

Cordeiro, H. T. D. (2012). Perfis de carreira da geração Y. Master’s Dissertation, Faculdade de Economia, Administração e Contabilidade, University of São Paulo, São Paulo. Doi: 10.11606/D.12.2012.tde07112012-201941. Retrieved 2020-05-11, from www.teses.usp.br

Dose, J. J. (1997). Work values: An integrative framework and illustrative application to organizational socialization. Journal of occupational and organizational psychology, 70(3), 219-240. Doi: https:// doi-org.ez22.periodicos.capes.gov.br/10.1111/j.20448325.1997.tb00645.x

Elizur, D. (1984). Facets of work values: A structural analysis of work outcomes. Journal of Applied Psychology, 69(3), 379-389. Doi: https://doi-org.ez22.periodicos.capes.gov.br/10.1037/0021-9010.69.3.379.

Estivalete, V. D. F. B., Löbler, M. L., de Andrade, T., \& Visentini, M. S. (2011). As implicações de gênero na hierarquia dos valores relativos ao trabalho. Revista Alcance, 18(3), 271-286. Doi: https://www. redalyc.org/articulo.oa?id=4777/477748595004

Faber, J. (2011). A Geração Z e a evolução das revistas científicas. Dental Press J Orthod, 16(4), 7.

Forbes. (2019). The ABCs Of Generations X, Y And Z. Retrieved August 10, 2019, from https://www.forbes. com/sites/forbeshumanresourcescouncil/2019/04/02/the-abcs-of-generations-x-y-and-z/\#6475f2a1672b 
Furucho, N. Y., Oswaldo, Y. C., Graziano, G. O., \& Elias Spers, V. R. (2015). Valores e Características Geracionais: Um Estudo em uma Instituição de Ensino Superior. Revista Brasileira de Marketing, 14(4), 492-501.

Grubb, V. M. (2016). Clash of the generations: Managing the new workplace reality. John Wiley \& Sons.

Hajdu, G., \& Sik, E. (2018). Age, Period, and Cohort Differences in Work Centrality and Work Values. Societies, 8(1), 11. Doi: http://dx.doi.org/10.3390/soc8010011

Hair Jr, J. F., Black, W. C., Babin, B. J., Anderson, R. E., \& Tatham, R. L. (2009). Análise multivariada de dados. Porto Alegre: Bookman

Holt, T., Burke-Smalley, LA e Jones, C. (2017), "An Empirical Investigation of Student Career Interests in Auditing Using the Big Five Model of Personality", Advances in Accounting Education: Teaching and Curriculum Innovations (Advances in Accounting Education, Vol. 20 ), Emerald Publishing Limited, pp. 1-31. Doi: https://doi.org/10.1108/S1085-462220170000020002

Kowske, B. J., Rasch, R., \& Wiley, J. (2010). Millennials' (lack of) attitude problem: An empirical examination of generational effects on work attitudes. Journal of Business and Psychology, 25(2), 265-279. Doi: https://doi.org/10.1007/s10869-010-9171-8

Lévy-Leboyer, C. (1994). A crise das motivações. Ed. Atlas

Maloni, M., Hiatt, M. S., \& Campbell, S. (2019). Understanding the work values of Gen Z business students. The International Journal of Management Education, 17(3), 100320. Doi: https://doi.org/10.1016/j. ijme.2019.100320

Mannheim, K., (1993). El problema de las generaciones. Reis, (62), 193-242. Doi: 10.2307/40183643

Moreira, S. A. S., \& de Araújo, B. F. V. B. (2018). Homens e Mulheres da Geração Y e suas Âncoras de Carreira. Desenvolvimento em Questão, 16(42), 621-650.

MOW International Research Team. (1987). The meaning of working. Academic Pr.

Ozkan, M., \& Solmaz, B. (2015). The changing face of the employees-generation $\mathrm{Z}$ and their perceptions of work (a study applied to university students). Procedia Economics and Finance, 26, 476-483. Doi: https://doi.org/10.1016/S2212-5671(15)00876-X

Parry, E., \& Urwin, P. (2011). Generational differences in work values: A review of theory and evidence. International journal of management reviews, 13(1), 79-96. Doi: http://dx.doi.org/10.111 $1 /$ j.1468-2370.2010.00285. x

Porto, J. B., \& Tamayo, A. (2003). Escala de valores relativos ao trabalho: EVT. Psicologia: teoria e pesquisa, 19(2), 145-152. Doi: https://doi.org/10.1590/S0102-37722003000200006

Porto, J. B, \& Tamayo, A. (2008). Valores do trabalho. In: M. M. M. Siqueira (Org.). Medidas do comportamento organizacional: ferramentas de diagnóstico e de gestão. Porto Alegre: Artmed.

Porto, J. B., \& Pilati, R. (2010). Escala Revisada de Valores Relativos ao Trabalho-EVT-R. Psicologia: Reflexão e Critica, 23(1), 73-82. Doi: https://doi.org/10.1590/S0102-79722010000100010

Potrich, A. C. G., Vieira, K. M., Estivalete, V. D. F. B., \& Andrade, T. D (2015). Valores relativos ao trabalho: testando a invariância e as diferenças de média entre os gêneros. Revista de Administração FACES Journal, 14(4), 44-64. Doi: https://doi.org/10.21714/1984-6975FACES2015V14N4ART2647

Revista Exame. (2017) A Geração Z e a conexão 24 horas por dia. Retrieved August 10, 2019, from https:// exame.abril.com.br/tecnologia/a-geracao-z-e-a-conexao-24-horas-por-dia/ 
Revista Exame. (2018). Saiba como gerenciar profissionais da geração Z. Retrieved August 10, 2019, from https://exame.abril.com.br/negocios/saiba-como-gerenciar-profissionais-da-geracao-z/

Roe, R. A., \& Ester, P. (1999). Values and work: Empirical findings and theoretical perspective. Applied psychology, 48(1), 1-21.

Ros, M., Schwartz, S. H., \& Surkiss, S. (1999). Basic individual values, work values, and the meaning of work. Applied psychology, 48(1), 49-71.

Santos Neto, E. D, \& Franco, E. S. (2010). Os professores e os desafios pedagógicos diante das novas gerações: considerações sobre o presente e o futuro. Revista de Educação do COGEIME, 19(36), 9-25. Doi: http://dx.doi.org/10.15599/0104-4834/cogeime.v19n36p9-25

Schwartz, S. H. (2006). Basic human values: An overview. Recuperado de http://www. yourmorals. org/schwartz.

Silva, M. R. M. S., Mendonça, H., \& Zanini, D. S. (2010). Diferenças de gênero e valores relativos ao trabalho. Paidéia (Ribeirão Preto), 20(45), 39-45. Doi: https://dx.doi.org/10.1590/S0103863X2010000100006

Smola, K.W., \& Sutton, C. D. (2002). Generational differences: Revisiting generational work values for the new millennium. Journal of Organizational Behavior: The International Journal of Industrial, Occupational and Organizational Psychology and Behavior, 23(4), 363-382. Doi: https://doi. org/10.1002/job.147

Twenge, J. M., \& Campbell, S. M. (2008). Generational differences in psychological traits and their impact on the workplace. Journal of managerial psychology, 23(8), 862-877. Doi: https://doi. org/10.1108/02683940810904367

Twenge, J. M., Campbell, S. M., Hoffman, B. J., \& Lance, C. E. (2010). Generational differences in work values: Leisure and extrinsic values increasing, social and intrinsic values decreasing. Journal of management, 36(5), 1117-1142. Doi: https://doi.org/10.1177/0149206309352246

Twenge, J. M., Campbell, W. K., \& Freeman, E. C. (2012). Generational differences in young adults' life goals, concern for others, and civic orientation, 1966-2009. Journal of personality and social psychology, 102(5), 1045-1062. Doi: https://doi.org/10.1037/a0027408

Weller, W. (2010). A atualidade do conceito de gerações de Karl Mannheim. Sociedade e Estado, 25(2), 205-224. Doi: https://doi.org/10.1590/S0102-69922010000200004

Zanelli, J. C., Borges-Andrade, J. E., \& Bastos, A. V. B. (2014). Psicologia, Organizações e Trabalho no Brasil-2. AMGH editora. 